\title{
A New Slip Length Model for Enhanced Water Flow Coupling Molecular Interaction, Pore Dimension, Wall Roughness, and Temperature
}

\author{
Lei Li, ${ }^{1,2}$ Yuliang Su $\mathbb{D}^{1,2}$ Han Wang, ${ }^{1,2}$ Guanglong Sheng, ${ }^{3}$ and Wendong Wang ${ }^{1,2}$ \\ ${ }^{1}$ Key Laboratory of Unconventional Oil \& Gas Development (China University of Petroleum (East China)), Ministry of Education, \\ Qingdao 266580, China \\ ${ }^{2}$ School of Petroleum Engineering, China University of Petroleum (East China), Qingdao 266580, China \\ ${ }^{3}$ School of Petroleum Engineering, Yangtze University, Wuhan 430100, China
}

Correspondence should be addressed to Yuliang Su; suyuliang@upc.edu.cn

Received 4 July 2019; Accepted 13 September 2019; Published 17 December 2019

Guest Editor: Wei Wei

Copyright (C) 2019 Lei Li et al. This is an open access article distributed under the Creative Commons Attribution License, which permits unrestricted use, distribution, and reproduction in any medium, provided the original work is properly cited.

\begin{abstract}
In this paper, a slip length model is proposed to analyze the enhanced flow based on the Hagen-Poiseuille equation. The model considers the multimechanisms including wall-water molecular interactions, pore dimensions, fractal roughness, and temperature. The increasing wall-water interactions result in the greater slip length and flow enhancement factor. The increased temperature enhances the kinetic energy of water molecules that leads to great surface diffusion coefficient and small work of adhesion. The wall roughness can decrease the slip length and flow enhancement factor in hydrophilic nanopores. This work studies the effects of multimechanisms on slip length and flow enhancement factor theoretically, which can accurately describe the liquid flow in nanopores.
\end{abstract}

\section{Introduction}

The transport behaviors of water confined in nanopores have attracted significant attention on science and engineering studies. The nanopores include carbon nanotubes (CNTs), boron nitride nanotubes (BNNTs), hydrophilic organic, and hydrophobic inorganic nanopores in shale reservoirs and other types of nanopores [1-3]. The results, measured or calculated by experimental studies, Molecular Dynamics Simulations (MDS), and theoretical studies, have shown that the enhanced water flow capacity in nanopores can be up to 1-5 orders of magnitude compared to that predicted by the no-slip Hagen-Poiseuille (HP) equation [4-6]. The behaviors of enhanced water flow are related to the boundary slip depending on many physical mechanisms, including pore wall-water molecular interactions (that can be expressed by surface wettability or contact angle) $[7,8]$, pore wall roughness [9-12], shear rate [13, 14], nanobubbles or gas films $[15,16]$, polarity of liquids [15-18], water viscosity $[19,20]$, temperature [21], pore dimensions [22], and pressure gradient [23]. In general, the boundary slip length is usually obtained by two methods: (1) microscopic slip length is observed by MDS; (2) macroscopic slip length is measured by experiments, and that cannot quantify the effect of each physical mechanism $[1,24]$. Therefore, it is of critical importance to analyze the effects of these physical mechanisms on water transport behaviors in nanopores based on incorporating experimental studies, MDS, and theoretical methods.

Many investigations have shown the flow enhancement factor of water flow in nanopores. The flow enhancement factor is defined as the ratio of the volume flux of water flow in nanopores to that calculated by the no-slip HP $[6,25,26]$. In addition, the flow enhancement factor strongly depends on the boundary slip length that the velocity at the wall is not zero [27]. Thomas and McGaughey [27] showed the slip length ranging from 105 to $30 \mathrm{~nm}$ of water transport in CNTs with $1.66-4.99 \mathrm{~nm}$ diameter by using MDS, and the enhancement factor ranged from 433 to 47 with an increasing CNT diameter. Then, with a diameter of $1 \mathrm{~nm}$ and $7 \mathrm{~nm}$, Falk et al. [28] found a slip length of $500 \mathrm{~nm}$ and $120 \mathrm{~nm}$ which was substantially smaller than the results measured by experiments of Majumder et al. [29]. In general, the wall-water system used in MDS is ideal that the pore wall is smooth. And there are usually some other factors that cannot be considered in MDS, such as the roughness and trapped gas at walls. Thus the slip length measured by experiments is about 14.6 times larger than that calculated by MDS [1]. In experimental studies, the slip length 
of $113-177 \mathrm{~nm}$ corresponded to the pore radius of $22 \mathrm{~nm}$ in nanopipes [30]. Secchi et al. [4] found 300-17 nm slip length for CNTs with diameters of 30-100 $\mathrm{nm}$ and the flow enhancement factor ranged from 2 to 24. Blake [7] extended Tolstoi's idea to establish the relationship between slip length and contact angle in theoretical studies. Later, Huang et al. [8] put forward a theoretical formula $L_{s}=C /(1+\cos \theta)^{2}$ to calculate slip length according to the contact angle, and the constant $C$ equals to 6 and 0.41 , which are used to fit the results of experiments and MDS respectively. Based on surface diffusion and work of adhesion, Mattia and Calabrò [31] proposed a theoretical model of water flow enhancement factor in CNTs. The values of the slip length and flow enhancement factor calculated by this model fitted well with those obtained by experimental and MDS results. These studies which were based on experimental studies, MDS, and theoretical methods have shown the different slip length and flow enhancement factor in nanopores with different conditions. Therefore, it is essential to understand the water transport behaviors in nanopores by an accurate calculation of slip length.

The slip length and flow enhancement factor can be measured or calculated by experiments, MDS, and theory methods respectively. However, these methods cannot quantitatively analyze the effects of different physical mechanisms comprehensively, such as surface wettability, surface roughness, shear rate, nanobubbles or gas films, polarity of liquids, liquid viscosity, temperature, pore radius, and pressure gradient [24]. The surface wettability can be described by the values of contact angle of the water droplet on the pore wall, and the contact angle is related to the liquid-solid molecular interactions. When the interactions are strong that the wall is hydrophilic, the contact angle is smaller than $90^{\circ}$ which leads to a smaller slip length $[8,32,33]$, and the slip length increases exponentially with an increasing contact angle [1]. From Wenzel and Cassie-Baxter equations, the wall roughness strongly affects the contact angle $[34,35]$. The roughness makes the effective contact angle smaller which results in the decrease in slip length when contact angle ranges from $0^{\circ}$ to $90^{\circ}$. When the contact angle ranges from $90^{\circ}$ to $180^{\circ}$, the effective contact angle increases as the roughness increases [36-39]. For the effects of gas films or trapped gas on the pore wall, Ruckenstein and Rajora [23] proposed that the water could flow on the wall with gas films, and the slip length would be larger considering gas films [40]. Because of the presence of trapped gas or pinned gas on rough wall, the contact angle increases leading to the larger slip length [41-43]. From experiments and MDS, the slip length also depends on shear rate, and the shear-depend slip is explicit in hydrophobic tubes [13,44]. In addition, the temperature also affects the slip length, Guo et al. [21] found that the slip length usually increased with a decreasing temperature by MDS methods. For the confined effects, the slip length monotonically decreases, and decreases rapidly first and then decreases slowly as the pore radius increases $[2,4,27]$. From the above discussion, there are various factors that can affect the slip length. However, the experiments cannot quantitatively analyze the microscopic mechanisms [1]. For the theoretical methods, Wu et al. [45] applied the slip length which depends on contact angle into the flow enhancement factor model without considering other mechanisms. Mattia and Calabrò [31] proposed calculation formula of the slip length that depends on pore radius, pore length, and solidliquid molecular interactions based on experiments and MDS. Nevertheless, their method cannot directly consider the changes of solid-liquid molecular interactions and the slip length is not relevant to pore length from results of Cui et al. [46] studies. Therefore, it is of great significance to clarify the effects of multimechanisms on the slip length through theoretical methods, or adopt new measured or simulation methods of experiments and MDS.

The theoretical formula of water flow enhancement factor is mostly obtained by the modified no-slip HP equation in which the viscosity of water is a constant value $[6,26,31,46]$. However, because of the wall-water molecular interactions, the water viscosity at the interface region is different from that in bulk region [47-49]. According to the results of experiments and MDS, the linear relationship between the water viscosity of interface and bulk region is calculated empirically based on the contact angle [1]. Thomas and McGaughey [27] proposed the effective viscosity that can be calculated by the viscosity and area of interface and bulk region. Then, the area of interface region can be calculated by the thickness of interface region which can be determined reasonably on the basics of MDS or theoretical studies. The results show that the thickness of interface region ranges from 0.7 to $1 \mathrm{~nm}$ [50-52]. Therefore, it is necessary to adopt the effective viscosity based on the water viscosity and thickness of interface region when calculating the enhancement factor.

In this paper, according to the no-slip HP equation, interfacial shear rate, surface diffusion coefficient, and work of adhesion, the calculation formula of slip length, incorporating multimechanisms including wall-water molecular interactions, pore dimensions, fractal roughness, and temperature, is established. First, the water flow enhancement model that considers boundary slip and the effective viscosity is derived. Then, the results of experimental studies and MDS are carried out to verify the proposed model. Finally, the effects of wall-water molecular interactions (contact angle), pore dimensions, fractal roughness, and temperature on slip length and flow enhancement factors are discussed.

\section{Mathematical Modeling}

In this section, the slip length related to the interfacial shear rate, surface diffusion coefficient, and work of adhesion is properly described, that depends on wall-water molecular interactions (contact angle), pore dimensions, fractal wall roughness, temperature, and effective viscosity. The surface diffusion coefficient is related to Fick's diffusion coefficient and thermodynamic factor. The work of adhesion is related to the liquid surface tension and temperature. Furthermore, the flow enhancement factor model is established based on the slip length and no-slip HP equation.

2.1. Boundary Slip Length. The water velocity at the pore wall strongly depends on the wall-water molecular interactions which leads to the slip length and changing viscosity of interface region [2]. In addition, the actual water velocity 


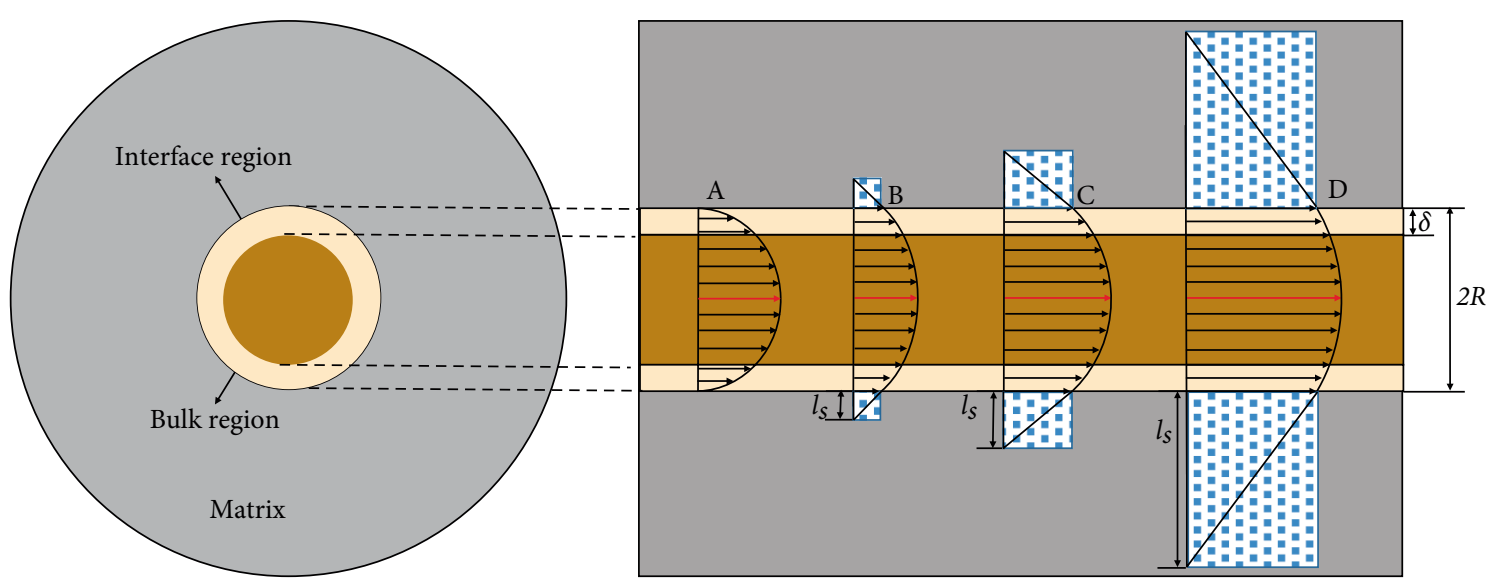

FIGURE 1: Schematic representation of water velocity profile in nanopores considering different wall-water molecular interactions.

considering boundary slip and water viscosity of interface region can be modified by the no-slip HP equation [31]. The water velocity profile in nanopores with different wall-water molecular interactions (contact angle) is shown in Figure 1. The slip length cannot be negative with contact angle ranging from $0^{\circ}$ to $180^{\circ}$. When the contact angle is close to $0^{\circ}$, the slip length will approach 0 . With different wall-water molecular interactions, the water viscosity is different that results in different values of maximum velocity.

As shown in section $\mathrm{A}$, the velocity without considering boundary slip and effective viscosity can be calculated by no-slip HP equation. From B to D, the velocity can be calculated by correctional HP equation considering a decreasing wall-water molecular interactions (an increasing contact angle) and water viscosity of interface region. The red arrows indicate maximum velocity, where $l_{s}$ is slip length; $\delta$ is thickness of interface region; $R$ is pore radius.

As shown in section A of Figure 1, the no-slip HP equation that describes the water velocity in nanopores with no velocity at the pore wall is given by:

$$
v_{H P}(r)=\frac{R^{2}-r^{2}}{4 \mu_{w b}} \frac{\partial p}{\partial z}
$$

where $v_{H P}(r)$ is water velocity with no-slip boundary, $R$ is pore radius, $r$ is pore radius of any position away from the pore center, $\mu_{b}$ is bulk viscosity of water, $\partial p / \partial z$ is pressure gradient.

Based on the no-slip HP equation, the water velocity considering boundary slip and changing viscosity of interface region can be expressed as $[46,48]$

$$
v(r)=\frac{R^{2}-r^{2}}{4 \mu_{\text {ave }}} \frac{\partial p}{\partial z}+v_{\text {slip }}
$$

where $v$ is velocity considering boundary slip, $\mu_{\text {ave }}$ is effective viscosity of water considering the changing viscosity of water, that can be calculated by water viscosity and area of bulk and interface region, $v_{\text {slip }}$ is the slip velocity at the pore wall.

The water molecules are strongly affected by the interactions from walls. Therefore, the water viscosity of interface region is different from that of bulk region. The critical thickness of the interface region can be reasonably determined as $0.7 \mathrm{~nm}$ from results of MDS and theoretical studies [50,53]. Based on the results from experiments and $\mathrm{MD}, \mathrm{Wu}$ et al. [1] proposed the relationship between the viscosity of the interface and bulk region, that is a function of the contact angle, and the relationship can be expressed as

$$
\mu_{i}=\left(-0.018 \theta_{0}+3.25\right) \mu_{w b},
$$

where $\mu_{i}$ is water viscosity of interface region, $\theta_{0}$ is contact angle of water droplet on the pore wall.

According to Thomas model of the spatial viscosity variation [27], the effective dynamic viscosity of water combining the viscosity and area of interface and bulk region can be expressed as

$$
\mu_{\text {ave }}(r)=\mu_{i} \frac{A_{i}(r)}{A_{t}(r)}+\mu_{w b} \frac{A_{w b}(r)}{A_{t}(r)},
$$

where $A_{i}(r), A_{b}(r)$, and $A_{t}(r)$ are area of interface, bulk and cross-section region of the nanopore, that can be calculated by pore radius and thickness of interface region, $A_{t}(r)=\pi R^{2}$, $A_{b}(r)=\pi r^{2}=\pi(R-\delta)^{2}, A_{i}(r)=\pi\left(R^{2}-(R-\delta)^{2}\right)$.

The boundary velocity $v_{\text {slip }}$ in Equation (2) is linearly related to the interfacial shear rate and slip length, therefore, based on the HP equation which can describe interfacial water transport with viscosity $\mu_{i}$, the boundary velocity can be expressed as $[31,54]$

$$
v_{s l i p}=-\left.l_{s}\left(\frac{\partial v_{H P}}{\partial r}\right)\right|_{r=R}=\frac{l_{s} R}{2 \mu_{i}} \frac{\partial p}{\partial z},
$$

where $l_{s}$ is the slip length, as shown in Figure 1.

From Tolstoi's model, Ruckenstein proposed a model to express the boundary velocity at pore wall based on surface diffusion coefficient, Boltzmann's constant and temperature, the boundary velocity can be expressed as [23]

$$
v_{s l i p}=\frac{D_{s}}{k_{B} T n_{L}} \frac{\partial p}{\partial z}
$$

where $D_{s}$ is the surface diffusion coefficient, $k_{B}$ is Boltzmann's constant, $T$ is temperature, $n_{L}$ is the number of molecules per unit volume at the interface region. 
According to expressions of boundary velocity of Equations (5) and (6), the boundary slip velocity at the wall can be expressed, the slip length can be derived as

$$
l_{s}=\frac{2 \mu_{i}}{R} \frac{D_{s}}{k_{B} T n_{L}} .
$$

According to Mattia's model, a low wall-water molecular interaction energy results in a low value of $k_{B} T n_{L}$ and a high value of $D_{s}$, and the constant value of $D_{s}=1 \times 10^{-9} \mathrm{~m}^{2} / \mathrm{s}$ and $W_{A}=144 \mathrm{~mJ} / \mathrm{m}^{2}$ was used to express the water flow in CNTs [31]. Later, Zhang et al. [6] and Cui et al. [46] also used constant value to simulate the oil transport in shale nanopores. However, different types of nanopores are with different wall-water interactions, even if the same types of nanopores are with small difference, the theoretical calculation of $k_{B} T n_{L}$ and $D_{s}$ for different wall-water interactions will be further discussed in the following sections.

2.1.1. Surface Diffusion. The surface diffusion coefficient is defined as the adsorbed water molecules jumping from one potential adsorption point to another $[23,55]$, that can be expressed as:

$$
D_{s}=\frac{\sigma^{2}}{4} \alpha=\frac{\sigma^{2}}{4} v_{0} e^{-E / k_{B} T},
$$

where $\sigma$ is the distance between two adsorption points, $\alpha$ is an effective jump frequency, $v_{0}$ is the vibrational frequency of water molecules at the interface region, $E$ is the potential energy barrier (the activation energy that molecules need to overcome jumping from one adsorption point to another).

Since the potential energy barrier is complicated, the surface diffusion coefficient is difficult to measure experimentally. The mean effective jump frequency depends on the coverage of adsorbed molecules at the wall $[56,57]$. Therefore, the surface diffusion coefficient taken to the coverage dependence is written as

$$
D_{s}=\frac{\sigma^{2}}{4} \Gamma(c),
$$

where $\Gamma(c)$ is a mean effective jump frequency, $c$ is fractional coverage of adsorbed molecules at interface region.

Based on the thermodynamic factor whose origin is the Kubo-Green formula, the semi-empirical formula is used to describe the relationship between surface diffusion and chemical surface diffusion coefficients that is given by $[56,57]$

$$
D_{\text {fick }}=\frac{\sigma^{2}}{4} \Gamma(c)\left(\frac{\partial\left(\chi / k_{B} T\right)}{\partial \ln c}\right)=D_{s}\left(\frac{\partial\left(\chi / k_{B} T\right)}{\partial \ln c}\right),
$$

where $\chi$ is chemical potential, $D_{\text {fick }}$ is chemical diffusion coefficient which is also called the Fick diffusion coefficient, that can be described by the Stokes-Einstein equation [58-60].

The Fick diffusion coefficient is affected by multifactors such as pore radius, molecular size and temperature, that can be described by the Stokes-Einstein equation [58, 61-63]

$$
D_{\text {fick }}=\frac{k_{B} T}{6 \pi \mu_{i} r_{A}},
$$

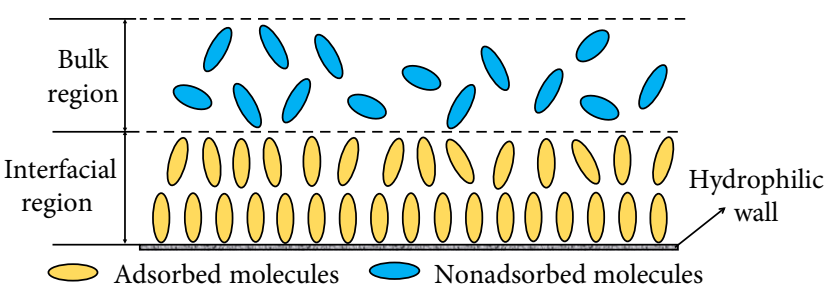

(a)

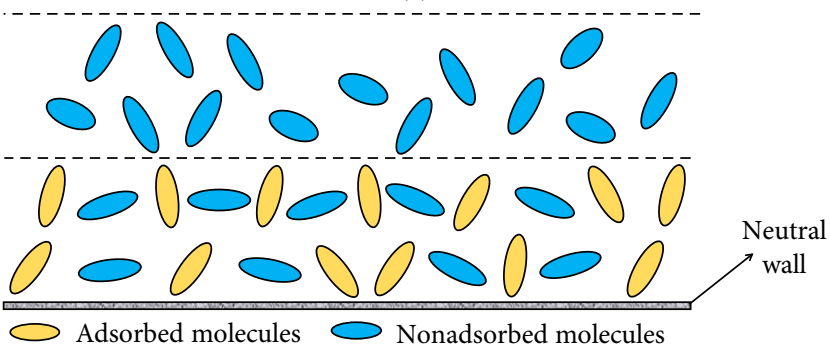

(b)

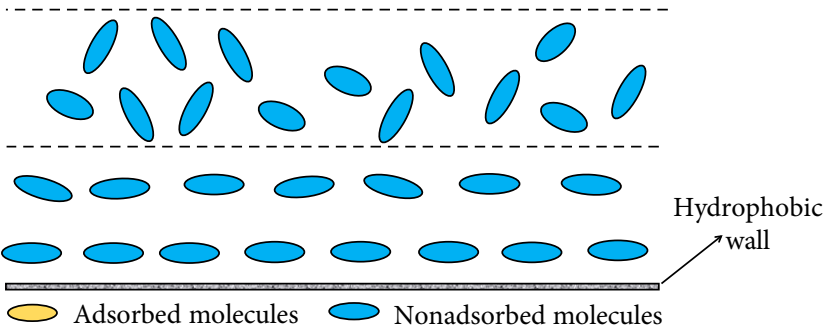

(c)

FIGURE 2: Distributions of water molecules with the different wettability of pore walls $[48,64]$.

where $r_{A}$ is molecule radius.

The thermodynamic factor $\left(\partial\left(\chi / k_{B} T\right) / \partial \ln c\right)$ can be written as $[56,57]$

$$
\left(\frac{\partial\left(\chi / k_{B} T\right)}{\partial \ln c}\right)=\frac{1}{1-c}\left(1+2 \frac{(1-\beta)}{\beta}\right) .
$$

The coefficient $\beta$ can be expressed as [56]

$$
\beta=\left(1-4 c(1-c)\left(1-e^{-w_{1} / k_{B} T}\right)\right)^{1 / 2},
$$

where $e^{-w_{1} / k_{B} T}$ is the interaction factors; $w_{1}$ is the interaction energy parameter, that can be approximated to $k_{B} T_{s}\left(w_{1}=k_{B} T_{s}\right)$ [56], and $T_{s}$ is the standard temperature $\left(T_{s}=273 \mathrm{~K}\right)$.

The parameter $c$ is defined as the fractional coverage of adsorbed molecules at the wall. The distribution state of water molecules at the interface region is strongly affected by the wall-water molecular interaction [64], as shown in Figure 2. From Figure 2(a), on the completely hydrophilic surface, the wall-water molecular interaction is strong which leads to a regular distribution of adsorbed water molecules on the wall. Hence the fractional coverage of adsorbed molecules is great and that is assumed to be close to 1. Figures 2(b) and 2(c) show the distribution of water molecules on the neutral and completely hydrophobic surface. With the decrease in wall-water interaction, the distribution of adsorbed molecules gradually becomes irregular that the fractional coverage becomes smaller. In this paper, it is assumed that 
the fractional adsorbed molecules depend on the wall-water molecular interactions (contact angle). The increasing contact angle depends on the decreasing wall-water molecular interactions, and the fraction coverage of adsorbed molecules decreases with an increasing contact angle. The fractional coverage of $0 \sim 1$ represents a contact angle of $180 \sim 0^{\circ}$. Based on assumption and normalization, the relationship between fractional coverage and contact angle can be described as

$$
c=\frac{1+\cos \theta_{0}}{2} .
$$

From Equations (8) to (14), the surface diffusion coefficient $D_{s}$ can be calculated by Fick's (second) law diffusion coefficient and thermodynamic factor, that is expressed as

$$
D_{s}=\frac{D_{f i c k}}{\left(\partial\left(\chi / k_{B} T\right) / \partial \ln c\right)}=\frac{k_{B} T}{6 \pi \mu_{i} r_{A}} \frac{1-c}{(1+2((1-\beta) / \beta))} .
$$

2.1.2. Work of Adhesion. In the previous work, Mattia and Calabrò [31] assumed $k_{B} T n_{L} L$ is the energy per unit surface of the water molecules at interface region that equaled to the work of adhesion, where $L$ is the pore length. However, $n_{L}$ is the number of water molecules per unit volume at the interface region, and the per unit surface can be approximated to the ratio of the per unit volume to the thickness of interface region. Then, the pore length in Mattia's model is replaced by thickness of interface region in our model. Therefore, in this paper, work of adhesion, which is defined as the interactions between water molecules and per unit surface of pore wall, is adopted based on thickness of interface region, that is expressed as

$$
W_{A}=k_{B} T n_{L} \delta,
$$

where $W_{A}$ is the work of adhesion; $\delta$ is thickness of interface region.

The work of adhesion of water with different wettability wall can be described in another way which is calculated according to the water surface tension and wall-water molecular interactions (contact angle), that is given by [65-67]

$$
W_{A}=\sigma_{1}\left(1+\cos \theta_{0}\right),
$$

where $\sigma_{1}$ is surface tension of water.

The surface tension of water usually decreases with an increasing temperature and vanishes at the critical point [68]. In this work, the Guggenheim equation is adopted to describe relationship between surface tension and temperature [69]

$$
\sigma_{1}=\sigma_{0}\left(1-T_{r}\right)^{11 / 9}
$$

where $\sigma_{0}$ is a temperature-independent constant (surface tension of water with temperature equaling to $0 \mathrm{~K}), T_{r}$ stands for reduced temperature which can be defined as the ratio of temperature to the critical temperature $\left(T_{r}=T / T_{c}\right), T_{c}$ is the critical temperature, and $T_{c}=647.3 \mathrm{~K}$ for water.

The temperature gradient of the surface tension for liquid is shown below [12]

$$
-\frac{d \sigma_{1}}{d T}=\frac{11}{9} \frac{\sigma_{0}}{T_{c}}\left(1-\frac{T}{T_{c}}\right)^{2 / 9},
$$

The temperature gradient of the surface tension for water is 0.16 [70]. Therefore, from Equation (19), the temperature-independent constant $(T=0 \mathrm{~K})$ is calculated, that $\sigma_{0}=84.74 \mathrm{~m} / \mathrm{Nm}$.

From Equations (7), (15), and (17), the slip length based on surface diffusion coefficient and work of adhesion is written as:

$$
\begin{aligned}
l_{s} & =\frac{2 \mu_{i}}{R} \frac{D_{s}}{k_{B} T n_{L}}=\frac{2 \mu_{i}}{R} \frac{\delta D_{s}}{W_{A}} \\
& =\frac{2 \mu_{i}}{R} \frac{\delta}{\sigma_{0}\left(1-T_{r}\right)^{11 / 9}\left(1+\cos \theta_{0}\right)} \frac{k_{B} T}{6 \pi \mu_{i} r_{A}} \frac{1-c}{(1+2(1-\beta) / \beta)} .
\end{aligned}
$$

2.2. Wall Roughness. From Equation (20), the slip length depends on the contact angle which is related to the wall-water molecular interactions, and the greater the interactions, the smaller the contact angle $[33,71]$. However, except the effects of wall-water molecular interactions, the contact angle is also affected by the wall roughness. When the contact angle ranges from $0^{\circ}$ to $90^{\circ}$, the contact angle will become smaller due to the roughness. On the contrary, when the contact angle ranges from $90^{\circ}$ to $180^{\circ}$, the contact angle will be greater due to the effects of pore wall roughness. That is, roughness will make the hydrophilic surface more hydrophilic, and make the hydrophobic surface more hydrophobic $[36-39,48]$. The apparent contact angle can be expressed by the Wenzel equation [34]

$$
\cos \left(\theta_{a p p}\right)=r_{a} \cos \left(\theta_{0}\right)
$$

where $\theta_{a p p}$ is the apparent contact angle affected by wall roughness, $r_{a}$ is a surface roughness factor that equals to the ratio of the actual area to project area.

With a rough surface, the factor $r_{a}$ is greater than $1\left(r_{a}>1\right)$. The pore surface with roughness is usually with the characteristics of fractal structures (or multihierarchical structures) $[45,48$, $72,73]$. Hence, the surface roughness factor and effective contact angle can be described in fractal form that are expressed as

$$
r_{a}=\left(\frac{l_{u}}{l_{l}}\right)^{D-2}
$$

$$
\cos \left(\theta_{a p p}\right)=\left(\frac{l_{u}}{l_{l}}\right)^{D-2} \cos \left(\theta_{0}\right),
$$

where $l_{u}$ and $l_{l}$ are upper and lower bound of rough elements, $D$ is the fractal dimension.

2.3. Flow Enhancement Factor. The flow enhancement factor is defined as the ratio of the volume flux calculated by the HP equation considering boundary slip to that predicated by noslip HP equation. From Equation (1) of no-slip HP equation, the volume flux of water is calculated by integrating $[31,74]$, that is expressed as

$$
Q_{H P}=\int_{0}^{R} 2 \pi r v_{H P}(r) d r=\int_{0}^{R} \frac{R^{2}-r^{2}}{4 \mu_{w b}} \frac{\partial p}{\partial z} 2 \pi r d r=\frac{\pi R^{4}}{8 \mu_{w b}} \frac{\partial p}{\partial z},
$$

where $Q_{H P}$ is volume flux calculated by no-slip HP equation. 
From Equations (2), (20), and (23), the volume flux considering boundary slip incorporating contact angle, pore radius, fractal roughness, temperature, and effective viscosity can be derived as

$$
\begin{aligned}
Q_{\text {slip }} & =\int_{0}^{R} v(r) 2 \pi r d r=\int_{0}^{R}\left(\frac{R^{2}-r^{2}}{4 \mu_{\text {ave }}}+\frac{\pi l_{s} R}{2 \mu_{i}}\right) \frac{\partial p}{\partial z} 2 \pi r d r \\
& =\left(\frac{\pi R^{4}}{8 \mu_{\text {ave }}}+\frac{\pi l_{s} R^{3}}{2 \mu_{i}}\right) \frac{\partial p}{\partial z},
\end{aligned}
$$

where $Q_{\text {slip }}$ is volume flux considering boundary slip.

Therefore, based on Equations (24) and (25), the flow enhancement factor is given by

$\varepsilon=\frac{Q_{\text {slip }}}{Q_{H P}}=\left(\frac{\mu_{w b}}{\mu_{\text {ave }}}+\frac{\mu_{w b}}{\mu_{i}} \frac{4 l_{s}}{R}\right)=\left(\frac{\mu_{w b}}{\mu_{\text {ave }}}+\frac{8 \mu_{i}}{R^{2}} \frac{10^{3} \delta D_{s}}{W_{A}} \frac{\mu_{w b}}{\mu_{i}}\right)$,

where $\varepsilon$ is the flow enhancement factor.

\section{Results and Discussion}

Based on the results obtained by experiments and MDS, the flow enhancement factor obtained by proposed model is validated. In addition, the effects of wall-water molecular interactions, pore dimensions, fractal roughness, and temperature on slip length and flow enhancement are discussed in this section.

3.1. Model Validation. Secchi et al. [4] and Thomas and McGaughey [27] have measured or calculated the flow enhancement factor through experiments and MDS, respectively. The parameters of contact angle, pore radius and temperature used in their studies and proposed model are shown in Table 1 . Because the roughness of pore wall is an uncontrollable factor in experiments, the wall roughness of CNTs and BNNTs used in experiments will have a small difference with different pore radius. During validation, we use the fractal dimension ranging from 2.017 to 2.023 to fit the experimental results, and the roughness scale $l_{u} / l_{l}$ equals to 10 . As shown in Figure 3(a), the light blue region is the enhancement factor obtained by proposed model with fractal dimension ranging from 2.017 to 2.023 . The results calculated by proposed model are fitted well with those of experiments. However, for the experimental enhancement factor of water flow in BNNTs, the results calculated by proposed model cannot fit it because the slip length in BNNTs is not controlled by the wetting properties [1].

The wall-water system which is established in MDS is ideal. Therefore, the value of fractal dimension used in proposed model is 2, which indicates the wall is smooth [27]. As shown in Figure 3(b), the flow enhancement factor calculated by the proposed model is fitted well with the simulation data of MDS. The results of the proposed model are fitted well with the results of experiments and MDS. Thus the proposed model is validated, and that can be used to study the liquid flow behaviors in nanopores.

\begin{tabular}{|c|c|c|c|}
\hline \multicolumn{2}{|c|}{ Experiment data verification } & \multicolumn{2}{|c|}{ MDS data verification } \\
\hline Parameter & Value & Parameter & Value \\
\hline Contact angle, $\theta$ & $155^{\circ}[4]$ & $\begin{array}{c}\text { Contact angle } \\
\theta\end{array}$ & $151^{\circ}[27]$ \\
\hline $\begin{array}{l}\text { Roughness } \\
\text { scale, } l_{u} / l_{l}\end{array}$ & 10 & $\begin{array}{c}\text { Roughness } \\
\text { scale, } l_{u} / l_{l}\end{array}$ & 10 \\
\hline $\begin{array}{l}\text { Fractal } \\
\text { dimension, } D\end{array}$ & $2.017 \sim 2.023$ & $\begin{array}{c}\text { Fractal } \\
\text { dimension, } D\end{array}$ & 2 \\
\hline Temperature, $T$ & $298.15 \mathrm{~K}[4]$ & Temperature, $T$ & $298 \mathrm{~K}[27]$ \\
\hline Pore radius, $R$ & $\begin{array}{c}15 \sim 50 \mathrm{~nm} \\
{[4]}\end{array}$ & Pore radius, $R$ & $\begin{array}{c}1.66 \sim 4.99 \mathrm{~nm} \\
{[27]}\end{array}$ \\
\hline $\begin{array}{l}\text { Thickness of } \\
\text { interface region, } \\
\delta\end{array}$ & $0.7 \mathrm{~nm}[50]$ & $\begin{array}{l}\text { Thickness } \\
\text { of interface } \\
\text { region, } \delta\end{array}$ & $0.7 \mathrm{~nm}[50]$ \\
\hline
\end{tabular}

TABLE 1: Basic parameters used in the proposed model.

3.2. Effects of Wall-Water Molecular Interactions. Figure 4 shows the dependence of the slip length and flow enhancement factor on contact angle with different pore radii. As shown in Figure 4(a), the slip length increases with the increase in contact angle at different radii. When the contact angle is close to $0^{\circ}$ which represents the strong wall-water interactions, the slip length can be reduced by five orders of magnitude. In contrast, when the contact angle equals to $179^{\circ}$ which represents the small interactions, the slip length can be increased up to five orders of magnitude. As shown in Figure 4(b), with a small contact angle, the flow enhancement factor can be decreased by one order of magnitude. The great effective viscosity and small slip length result in the small velocity and then weaken the water flow ability, as shown in section B of Figure 1. When the contact angle is close to $179^{\circ}$, the flow enhancement factor can be increased up to seven orders of magnitude. And the reason is that the small wall-water molecular interactions can lead to a small effective viscosity and a great slip length, as shown in section $\mathrm{D}$ of Figure 1 . In a word, the flow enhancement factor is $0.4 \sim 10^{7}$ times higher than that of noslip HP equation with contact angle ranging from 0 to $179^{\circ}$. Therefore, the slip length and flow enhancement factor are strongly affected by the wall-water molecular interactions with different pore radii, and the results are consistent with the enhanced water flow in hydrophobic CNTs $[1,4]$.

3.3. Effects of Pore Dimensions and Temperature. The dependence of the slip length and flow enhancement factor on pore radius with different contact angle is shown in Figure 5. As shown in Figure 5(a), the slip length strongly depends on the pore radius, especially with a small pore radius. Hence, the slip length decreases rapidly first and then decreases slowly as pore radius increases. This trend is consistent with the results of experiments, MDS, and theories $[4,6,27]$. As shown in Figure 5(b), when the pore radius is smaller than $15 \mathrm{~nm}$, the flow enhancement factor changes greatly with a small difference of pore radius. The reason is that the strong dependence of the structure and dynamics of the confined water on the dimension.

Figure 6 shows the effects of the temperature on the slip length and flow enhancement factor. The increasing 


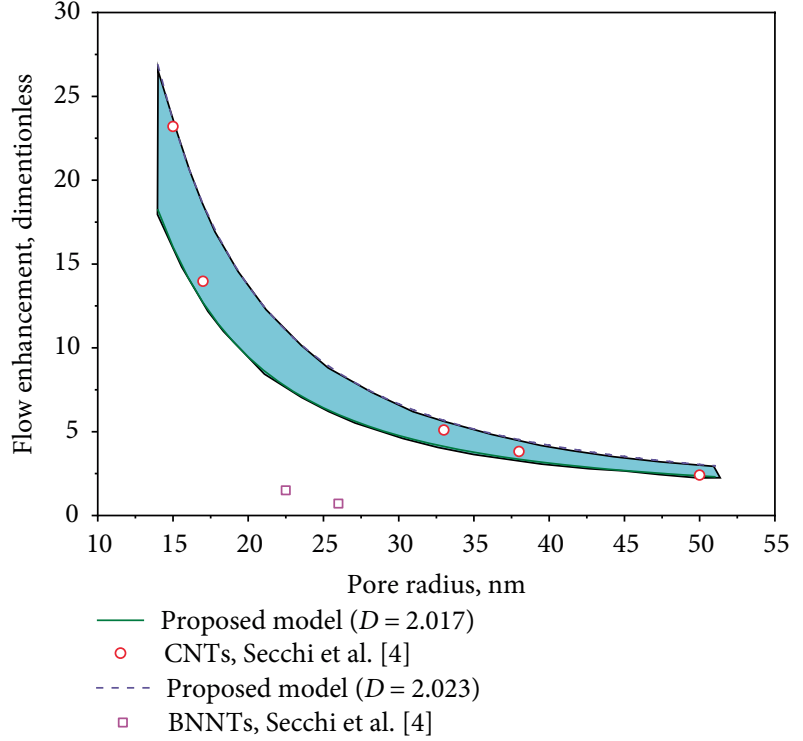

(a)

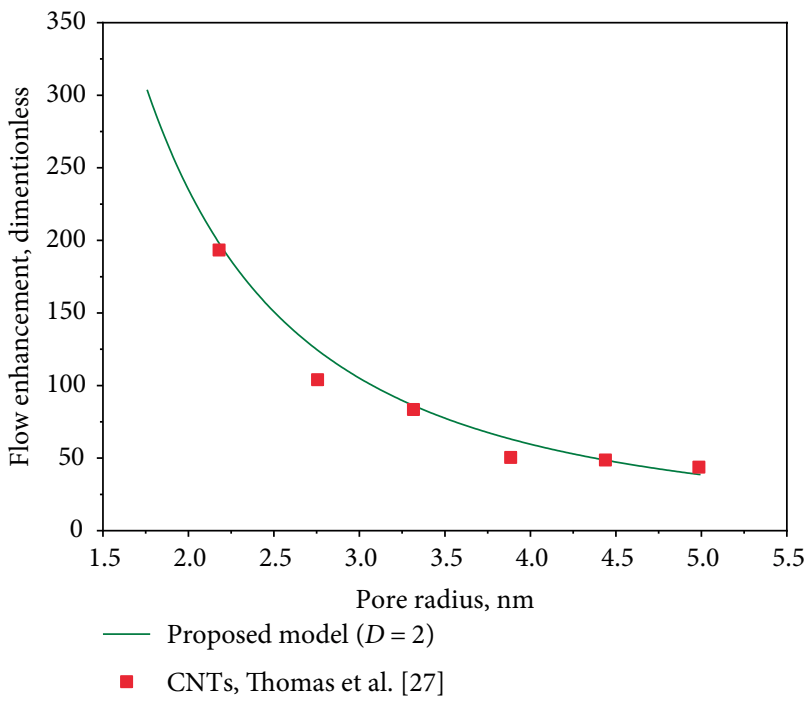

(b)

Figure 3: Comparison of flow enhancement factor obtained by experiments, MDS and proposed model. (a) comparison of flow enhancement factor of proposed model and experiment [4], the light blue region is the enhancement factor of proposed model with fractal dimension ranging from 2.017 to 2.023; (b) comparison of flow enhancement factor calculated by proposed model and MDS [27].

temperature can enhance the kinetic energy of water molecules, and decrease the energy barrier for water molecules to diffuse from one adsorption point to another. Therefore, the surface diffusion coefficient will increase. Additionally, because the increasing temperature can decrease surface tension, the work of adhesion will decrease. Thus, the slip length and flow enhancement factor increase with an increasing temperature due to the increasing surface diffusion coefficient and the decreasing work of adhesion.

3.4. Effects of Fractal Roughness. Figure 7 shows that the slip length and flow enhancement factor vary with the contact angle with different fractal dimensions. The ratio

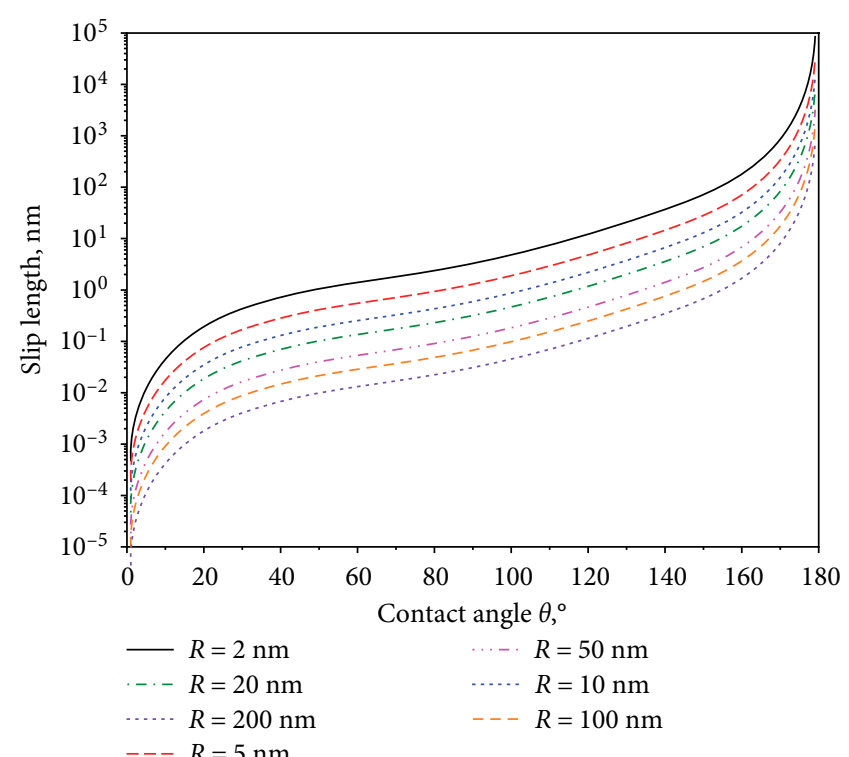

(a)

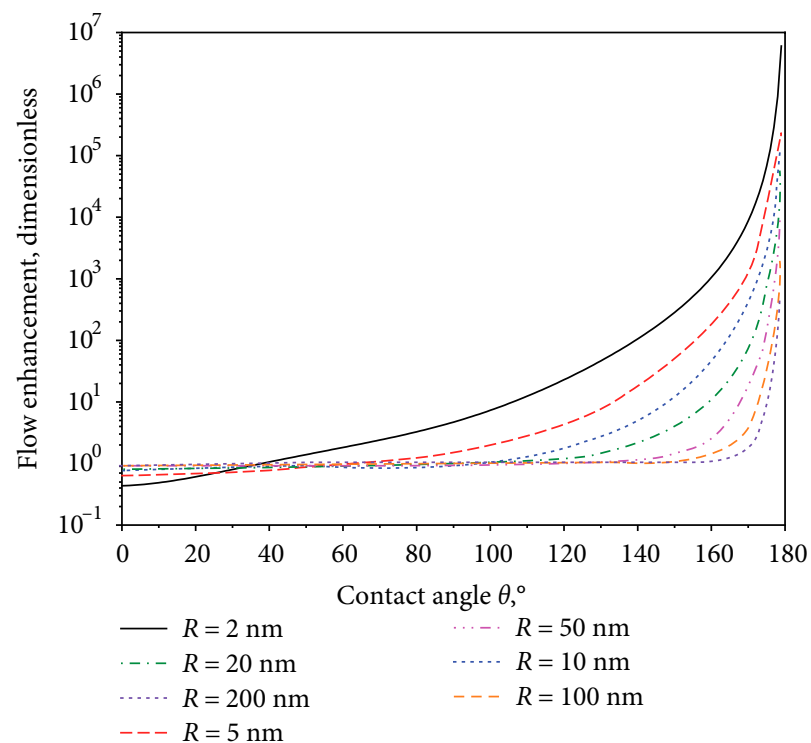

(b)

FIGURE 4: The effect of wall-water molecular interactions on the slip length and flow enhancement factor with different pore radii. (a) Slip length versus contact angle with different pore radii. (b) Flow enhancement versus contact angle with different pore radii.

of the upper to lower bound is set as $10\left(l_{u} / l_{l}=10\right)$ as an example. As shown in Figure 7(a), the slip length increases with an increasing fractal dimension when the contact angle is greater than $90^{\circ}$. The reason is that compared to the contact angle of water droplet on smooth surface (the contact angle is represented by $\theta_{0}$ ), the apparent contact angle is larger due to the effects of wall roughness (the contact angle is represented by $\theta_{a p p}$ ), and as the fractal dimension increases, the apparent contact angle $\theta_{a p p}$ increases with a constant contact angle $\theta_{0}$. Therefore, with a larger fractal dimension which represents the larger effective contact angle, the slip length will become greater. In addition, as the contact angle increases, the slip length gradually reaches the maximum 


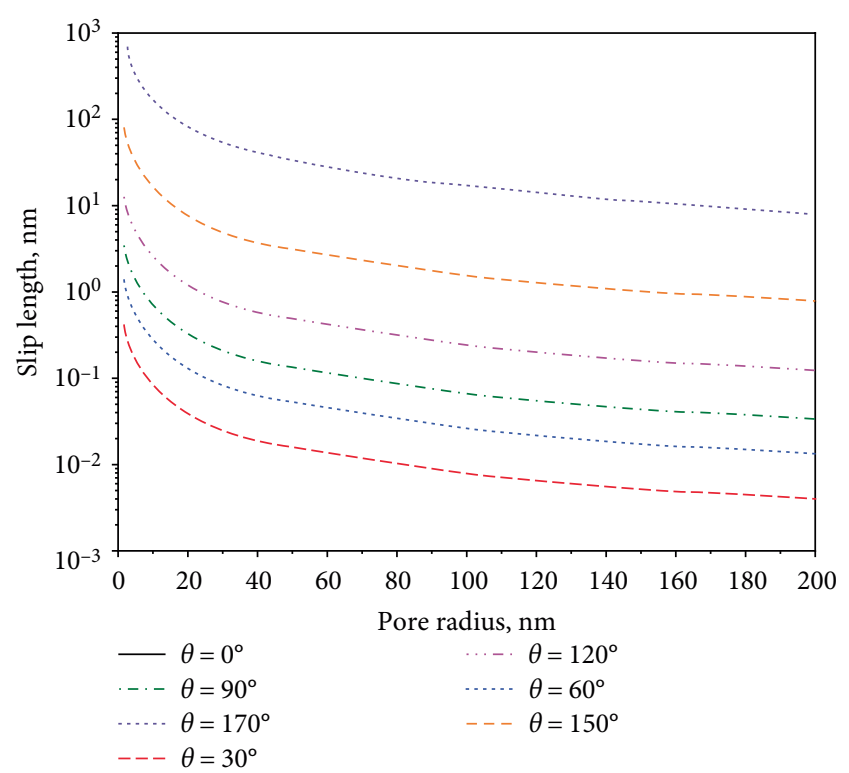

(a)

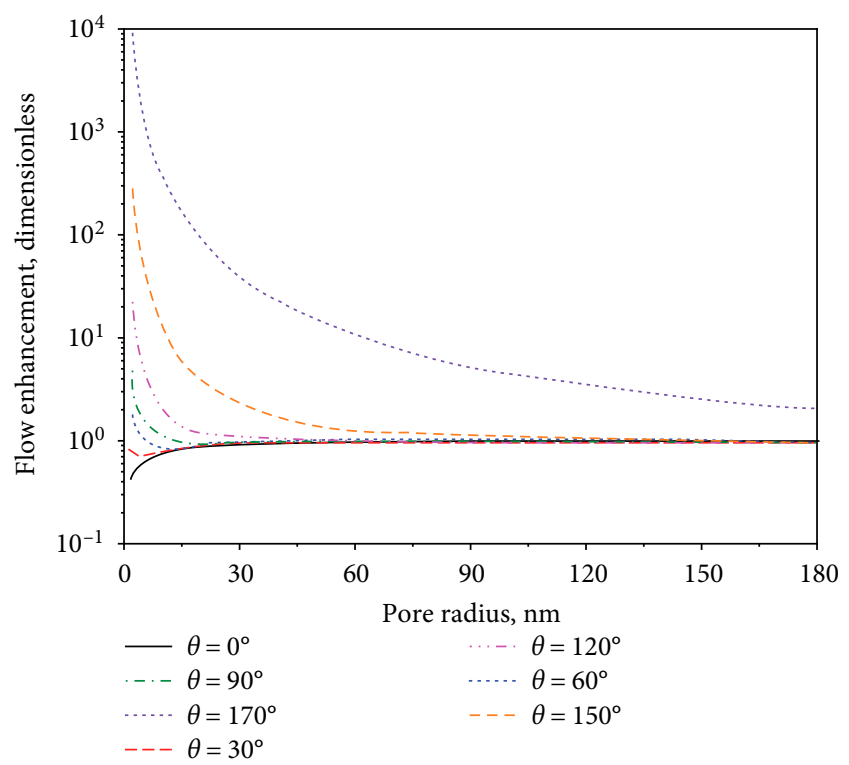

(b)

FIgURE 5: The effect of pore radius on the slip length and flow enhancement factor with different contact angle. (a) Slip length versus pore radius with different contact angle. (b) Flow enhancement versus pore radius with different contact angle.

because the apparent contact angle is close to $180^{\circ}$. For example, when fractal dimension equals to $2.1(D=2.1)$, the apparent contact angle will approach $180^{\circ}\left(\theta_{\text {app }} \approx 180^{\circ}\right)$ because of the roughness when the contact angle is greater than $142^{\circ}\left(\theta_{0}>142^{\circ}\right)$. At this time, the slip length approaches maximum. On the contrary, when contact angle is smaller than $90^{\circ}$, the apparent contact angle is smaller than contact angle due to the roughness. The greater the fractal dimension, the smaller the apparent contact angle. Therefore, the slip length decreases as the fractal dimension increases. As shown in Figure 7(b), when the contact angle is smaller than $90^{\circ}$, the flow enhancement factor is a constant value. The reason

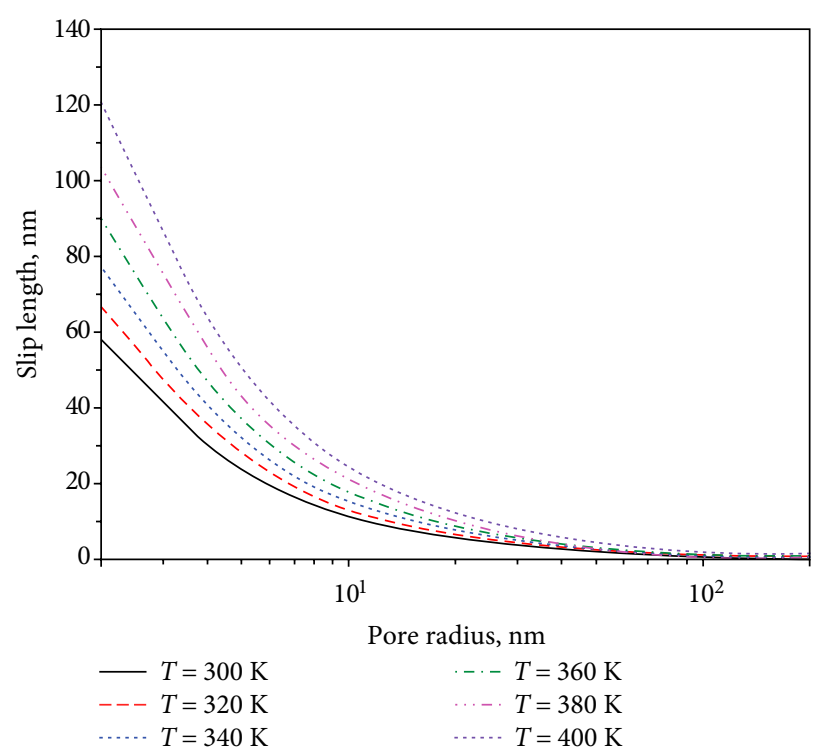

(a)

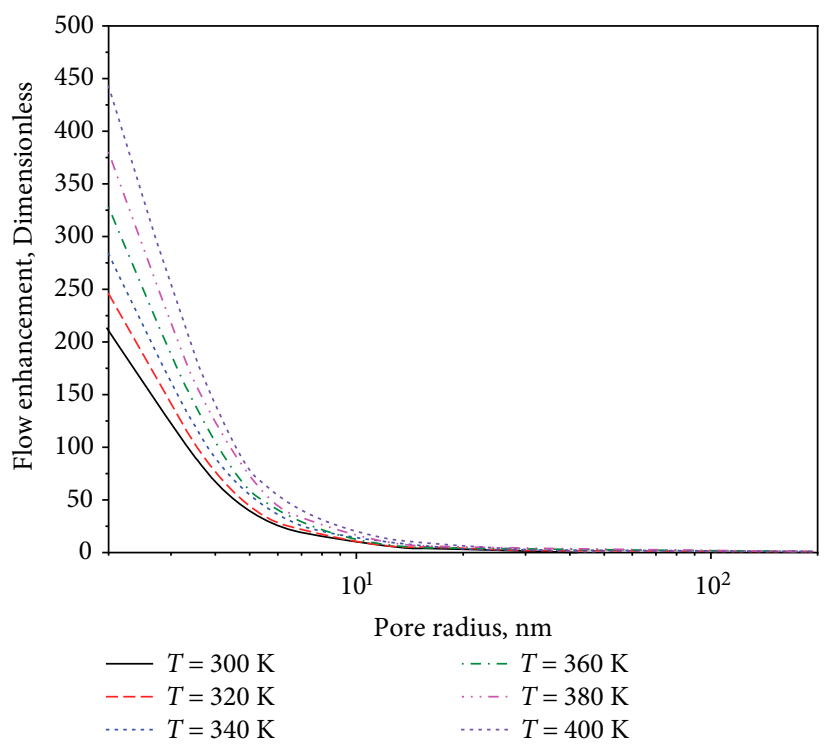

(b)

FIGURE 6: The effects of pore radius on the slip length and flow enhancement factor with different temperature, and the contact angle used in proposed model is $150^{\circ}$. (a) Slip length versus pore radius with different temperature. (b) Flow enhancement versus pore radius with different temperature.

is that the apparent contact angle approaches to $0^{\circ}$ because of the wall roughness. Then the flow enhancement factor increases gradually and approach maximum as contact angle increases. In addition, the flow enhancement factor decreases with an increasing fractal dimension. When the contact angle ranges from $90^{\circ}$ to $180^{\circ}$, the flow enhancement factor increases with an increasing fractal dimension.

In conclusion, the wall roughness can enhance the hydrophobicity of hydrophobic surface and the hydrophilicity of hydrophilic surface, respectively. Therefore, the slip length and flow enhancement will increase and decrease respectively in hydrophobic and hydrophilic nanopores because of the wall roughness. 


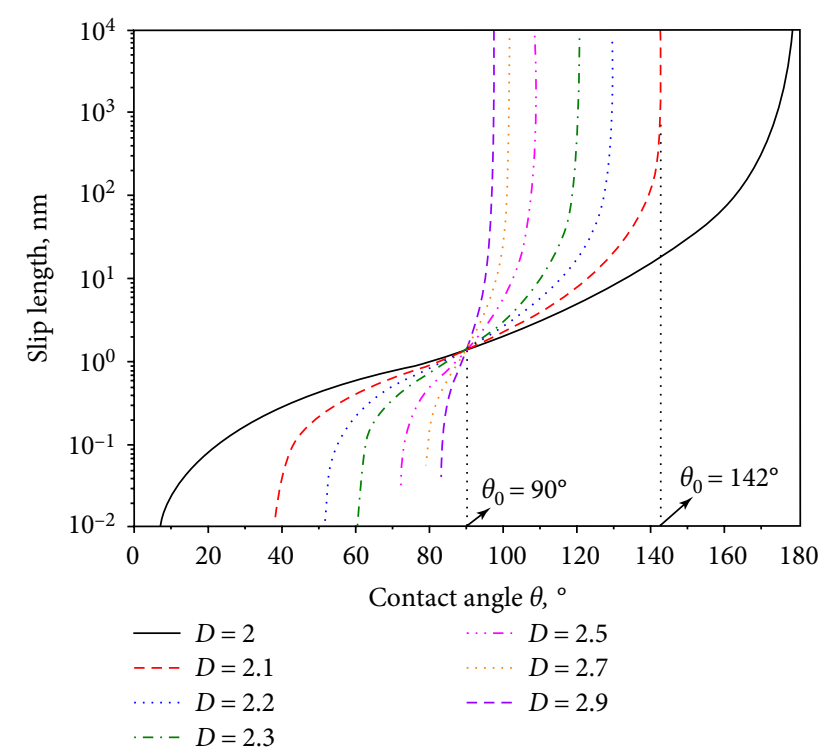

(a)

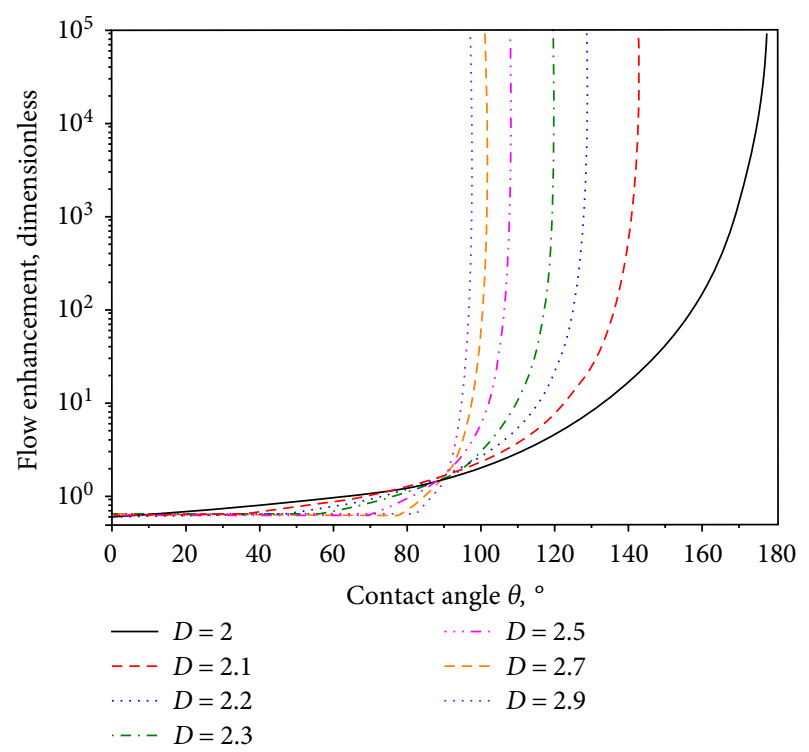

(b)

Figure 7: The effects of contact angle on the slip length and flow enhancement factor with different fractal dimensions, and the pore radius used in proposed model is $5 \mathrm{~nm}$. (a) Relationship between slip length and fractal dimensions; (b) relationship between flow enhancement and fractal dimensions.

\section{Conclusion}

Based on the no-slip HP equation, a slip length model is proposed to analyze the enhanced flow and study the transport behaviors. The proposed model takes into account the multimechanisms of wall-water molecular interactions, pore dimensions, fractal roughness, and temperature. The proposed model is validated with the results of experiments and MDS. In addition, the effects of contact angle, pore radius, fractal dimension, and temperature on slip length and flow enhancement factor are discussed. Our results have demonstrated that the slip length and flow enhancement factor greatly depend on these multimechanisms.
The slip length and flow enhancement factor increase with an increasing contact angle which represents the increasing wall-water molecular interactions. The flow enhancement factor is $0.4 \sim 10^{7}$ times higher than that predicted by no-slip HP equation with contact angle ranging from 0 to $179^{\circ}$. Because of structure and dynamics of the confined effects, the slip length and flow enhancement factor increase as pore radius decreases, especially for pore radius smaller than $15 \mathrm{~nm}$. The slip length and flow enhancement factor will have a great change with a small difference of pore radius. The increasing temperature can enhance the kinetic energy of water molecules, that leads to a great surface diffusion coefficient and small work of adhesion. Hence, the flow enhancement factor will become greater with an increasing temperature. In addition, through influencing the contact angle, the wall roughness can decrease or increase the slip length and flow enhancement factor in hydrophilic and hydrophobic nanopores, respectively. This work comprehensively analyzes the effects of multimechanisms on slip length and flow enhancement factor. It is meaningful for us to clarify the liquid transport behaviors in nanopores and effectively combine studies of experiments, MDS and theory.

\section{Data Availability}

The data used to support the findings of this study are included within the article. All the data used in the figures was derived from the euqations presented in this article.

\section{Conflicts of Interest}

The authors declare that they have no conflicts of interest.

\section{Nomenclature}

$A_{i}: \quad$ Area of interface region $\left(\mathrm{nm}^{2}\right)$

$A_{t}$ : Area of cross-section region of the pore $\left(\mathrm{nm}^{2}\right)$

$A_{w b}: \quad$ Area of bulk region $\left(\mathrm{nm}^{2}\right)$

$c: \quad$ Fractional coverage (dimensionless)

$D: \quad$ Fractal dimension (dimensionless)

$D_{\text {fick: }}$ : Chemical diffusion coefficient $\left(\mathrm{m}^{2} / \mathrm{s}\right)$

$D_{s}: \quad$ Surface diffusion coefficient $\left(\mathrm{m}^{2} / \mathrm{s}\right)$

$E: \quad$ Potential energy barrier $(\mathrm{J})$

$k_{B}: \quad$ Boltzmann's constant $(\mathrm{J} / \mathrm{K})$

$L: \quad$ Pore length $(\mathrm{nm})$

$l_{l}$ : $\quad$ Lower bound $(\mathrm{nm})$

$l_{s}: \quad$ Slip length $(\mathrm{nm})$

$l_{u}: \quad$ Upper bound $(\mathrm{nm})$

$n_{L}: \quad$ Number of molecules per unit volume $\left(1 / \mathrm{m}^{3}\right)$

$\partial p / \partial z: \quad$ Pressure gradient $(\mathrm{MPa} / \mathrm{m})$

$Q_{H P}: \quad$ Volume flux calculated by no-slip HP equation $\left(\mathrm{m}^{3} / \mathrm{s}\right)$

$Q_{\text {slip }}: \quad$ Volume flux considering boundary slip $\left(\mathrm{m}^{3} / \mathrm{s}\right)$

$r: \quad$ Position away from the pore center $(\mathrm{nm})$

$R: \quad$ Pore radius $(\mathrm{nm})$

$r_{a}: \quad$ Roughness factor (dimensionless)

$r_{A}: \quad$ Water molecule radius $(\mathrm{nm})$ 
$T: \quad$ Temperature (K)

$T_{c}: \quad$ Critical temperature (K)

$T_{r}$ : $\quad$ Reduced temperature (dimensionless)

$T_{s}: \quad$ Stand temperature $(\mathrm{K})$

$v: \quad$ Velocity considering boundary slip $(\mathrm{m} / \mathrm{s})$

$v_{0}: \quad$ Vibrational frequency of water molecules $(1 / \mathrm{s})$

$v_{H P}: \quad$ Velocity with no-slip boundary $(\mathrm{m} / \mathrm{s})$

$v_{\text {slip }}: \quad$ Water velocity at the wall $(\mathrm{m} / \mathrm{s})$

$w_{1}: \quad$ Interaction energy parameter $(\mathrm{J})$

$W_{A}: \quad$ Work of adhesion $\left(\mathrm{mJ} / \mathrm{m}^{2}\right)$

$\sigma_{1}: \quad$ Surface tension of water $(\mathrm{mN} / \mathrm{m})$

$\sigma_{0}$ : Temperature-independent constant $(\mathrm{mN} / \mathrm{m})$

$\sigma: \quad$ Distance between two adsorption points (nm)

$\alpha: \quad$ Effective jump frequency $(1 / \mathrm{s})$

$\delta: \quad$ Thickness of interface region $(\mathrm{nm})$

$\theta_{0}: \quad$ Contact angle of water droplet on the wall $\left({ }^{\circ}\right)$

$\theta_{a p p}: \quad$ Apparent contact angle $\left(^{\circ}\right)$

$\mu_{\text {ave }}: \quad$ Effective viscosity of water $(\mathrm{mPa} \cdot \mathrm{s})$

$\mu_{w b}: \quad$ Bulk viscosity of water ( $\mathrm{mPa} \cdot \mathrm{s}$ )

$\mu_{i}: \quad$ Water viscosity of interface region ( $\mathrm{mPa} \cdot \mathrm{s}$ )

$\varepsilon: \quad$ Flow enhancement factor (dimensionless)

$\chi: \quad$ Chemical potential (J)

$\Gamma: \quad$ Mean effective jump frequency $(1 / \mathrm{s})$

$\beta$ : Coefficient depending on fractional coverage (dimensionless).

\section{Acknowledgments}

This study was supported by the Province Natural Science Foundation of Shandong (ZR2018BEE018), National Natural Science Foundation of China (51904324, 51974348, and 51804328), the Major National Research and Development Projects of China (2017ZX05049 and 2017ZX05009), China Postdoctoral Science Foundation (2018M630813, 2019T120616), the Fundamental Research Funds for the Central Universities (18CX02170A), and the Postdoctoral Applied Research Project Foundation of Qingdao city (BY201802003).

\section{References}

[1] K. Wu, Z. Chen, J. Li, X. Li, J. Xu, and X. Dong, "Wettability effect on nanoconfined water flow," Proceedings of the National Academy of Sciences of the United States of America, vol. 114, no. 13, pp. 3358-3363, 2017.

[2] S. K. Kannam, B. D. Todd, J. S. Hansen, and P. J. Daivis, "How fast does water flow in carbon nanotubes?" The Journal of Chemical Physics, vol. 138, no. 9, p. 094701, 2013.

[3] Y. Su, H. Wang, G. Sheng, W. Wang, Q. Zhang, and S. Zhan, "A model for gas transport in organic matter with isolated pores in shale gas reservoirs," Journal of Natural Gas Science and Engineering, vol. 57, pp. 178-188, 2018.

[4] E. Secchi, S. Marbach, and A. Nigues, D. Stein, A. Siria, and L. Bocquet, "Massive radius-dependent flow slippage in carbon nanotubes," Nature, vol. 537, no. 7619, pp. 210-213, 2016.

[5] H. Wang, Y. Su, W. Wang, G. Sheng, H. Li, and A. Zafar, "Enhanced water flow and apparent viscosity model considering wettability and shape effects," Fuel, vol. 253, pp. 1351-1360, 2019.

[6] Q. Zhang, Y. Su, W. Wang, M. Lu, and G. Sheng, "Apparent permeability for liquid transport in nanopores of shale reservoirs: coupling flow enhancement and near wall flow," International Journal of Heat and Mass Transfer, vol. 115, pp. 224-234, 2017.

[7] T. D. Blake, "Slip between a liquid and a solid: D. M. Tolstoi's (1952) theory reconsidered," Colloids and Surfaces, vol. 47, pp. 135-145, 1990.

[8] D. Huang, C. Sendner, and D. Horinek, "Water slippage versus contact angle: a quasiuniversal relationship," Physical Review Letters, vol. 101, no. 22, p. 226101, 2008.

[9] T. M. Galea and P. Attard, "Molecular dynamics study of the effect of atomic roughness on the slip length at the fluidsolid boundary during shear flow," Langmuir, vol. 20, no. 8, pp. 3477-3482, 2004.

[10] A. Kucala, M. J. Martinez, Y. Wang, and D. R. Nobel, "The influence of interfacial slip on two-phase flow in rough pores," Water Resource Research, vol. 53, no. 8, pp. 7281-7295, 2017.

[11] S. Pasquier, M. Quintard, and Y. Davit, "Modeling flow in porous media with rough surfaces: effective slip boundary conditions and application to structured packings," Chemical Engineering Science, vol. 165, pp. 131-146, 2017.

[12] Z. Javidi, Z. Tarashi, A. Rostami, and H. Nazockdast, "Role of nanosilica localization on morphology development of HDPE/ PS/PMMA immiscible ternary blends," Express Polymer Letters, vol. 11, no. 5, pp. 362-373, 2017.

[13] C. Choi, K. Westin, and K. Breuer, "Apparent slip in hydrophilic and hydrophobic microchannels," Physics of Fluids, vol. 15, no. 10, p. 2897, 2003.

[14] M. Sun and C. Ebner, "Molecular dynamics study of flow at a fluid-wall interface," Physics Review Letters, vol. 69, no. 24, pp. 3491-3494, 1992.

[15] U.-C. Boehnke, T. Remmler, H. Motschmann, S. Wurlitzer, J. Hauwede, and T. M. Fischer, "Partial air wetting on solvophobic surfaces in polar liquids," Journal of Colloid and Interface Science, vol. 211, pp. 243-251, 1999.

[16] H. Hu, D. Wang, F. Ren, L. Bao, N. V. Priezjev, and J. Wen, "A comparative analysis of the effective and local slip lengths for liquid flows over a trapped nanobubble," International Journal of Multiphase Flow, vol. 104, pp. 166-173, 2018.

[17] J. Cho, B. Law, and F. Rieutord, "Dipole-dependent slip of newtonian liquids at smooth solid hydrophobic surfaces," Physical Review Letters, vol. 92, no. 16, p. 166102, 2004.

[18] N. K. Ranjit, G. C. Shit, and A. Sinha, "Transportation of ionic liquids in a porous micro-channel induced by peristaltic wave with Joule heating and wall-slip conditions," Chemical Engineering Sciences, vol. 171, pp. 545-557, 2017.

[19] N. Priezjev and S. Troian, "Molecular origin and dynamic behavior of slip in sheared polymer films," Physical Review Letters, vol. 92, no. 1, p. 018302, 2004.

[20] S. Lichter, A. Martini, and R. Snurr, "Liquid slip in nanoscale channels as a rate process," Physical Review Letters, vol. 98, no. 22, p. 226001, 2007.

[21] Z. Guo, T. Zhao, and Y. Shi, "Temperature dependence of the velocity boundary condition for nanoscale fluid flows," Physical Review E, vol. 72, no. 3, p. 036301, 2005.

[22] S. Kannam, B. Todd, and J. Hansen, "Slip length of water on graphene: limitations of non-equilibrium molecular dynamics 
simulations," The Journal of Chemical Physics, vol. 136, no. 2, p. $024705,2012$.

[23] E. Ruckenstein and P. Rajora, "On the no-slip boundary condition of hydrodynamics," Journal of Colloid and Interface Science, vol. 96, no. 2, pp. 488-491, 1983.

[24] B.-Y. Cao, J. Sun, M. Chen, and Z.-Y. Guo, "Molecular momentum transport at fluid-solid interfaces in mems/nems: a review," International Journal of Molecular Science, vol. 10, no. 11, pp. 4638-4706, 2009.

[25] J. S. Babu and S. P. Sathian, "The role of activation energy and reduced viscosity on the enhancement of water flow through carbon nanotubes," The Journal of Chemical Physics, vol. 134, no. 19, p. 194509, 2011.

[26] D. Mattia, H. Leese, and K. P. Lee, "Carbon nanotube membranes: From flow enhancement to permeability," Journal of Membrane Science, vol. 475, pp. 266-272, 2015.

[27] J. A. Thomas and A. J. H. McGaughey, "Reassessing fast water transport through carbon nanotubes," Nano Letters, vol. 8, no. 9, pp. 2788-2793, 2008.

[28] K. Falk, F. Sedlmeier, L. Joly, R. R. Netz, and L. Bocquet, "Molecular origin of fast water transport in carbon nanotube membranes: superlubricity versus curvature dependent friction," Nano letters, vol. 10, no. 10, pp. 4067-4073, 2010.

[29] M. Majumder, N. Chopra, R. Andrew, and B. J. Hinds, "Enhanced flow in carbon nanotubes," Nature, vol. 438, no. 7064, p. 44, 2005.

[30] M. Whitby, L. Cagnon, M. Thanou, and N. Quirke, "Enhanced fluid flow through nanoscale carbon pipes," Nano Letters, vol. 8, no. 9, pp. 2632-2637, 2008.

[31] D. Mattia and F. Calabrò, "Explaining high flow rate of water in carbon nanotubes via solid-liquid molecular interactions," Microfluid Nanofluid, vol. 13, no. 1, pp. 125-130, 2012.

[32] D. Ortiz-Young, H.-C. Chiu, S. Kim, K. Voïtchovsky, and E. Riedo, "The interplay between apparent viscosity and wettability in nanoconfined water," Nature Communications, vol. 4 , no. 1, p. 2482, 2013.

[33] Y. Xue, Y. Wu, X. Pei, H. Duan, Q. Xue, and F. Zhou, "How solid-liquid adhesive property regulates liquid slippage on solid surfaces?," Langmuir, vol. 31, no. 1, pp. 226-232, 2014.

[34] R. N. Wenzel, "Resistance of solid surfaces to wetting by water," Industrial and Engineering Chemisry Research, vol. 28, no. 8, pp. 988-994, 1936.

[35] A. B. D. Cassie and S. Baxter, "Wettability of porous surfaces," Transactions of the Faraday Society, vol. 40, pp. 546-551, 1944.

[36] F. De Nicola, P. Castrucci, M. Scarselli, F. Nanni, I. De Cacciotti, and M. Crescenzi, "Multi-fractal hierarchy of single-walled carbon nanotube hydrophobic coatings," Scientific Reports, vol. 5, no. 1, p. 8583, 2015.

[37] K. Cheng, B. Naccarato, K. J. Kim, and A. Kumar, “Theoretical consideration of contact angle hysteresis using surface-energyminimization methods," International Journal of Heat and Mass Transfer, vol. 102, pp. 154-161, 2016.

[38] S. Suzuki and K. Ueno, "Apparent contact angle calculated from a water repellent model with pinning effect," Langmuir, vol. 33, no. 1, pp. 138-143, 2017.

[39] R. Jain and R. Pitchumani, "Fractal model for wettability of rough surfaces," Langmuir, vol. 33, no. 28, pp. 7181-7190, 2017.

[40] O. I. Vinogradova, "Drainage of a thin liquid film confined between hydrophobic surfaces," Langmuir, vol. 11, no. 6, pp. 2213-2220, 1995.
[41] C. Cottin-Bizonne, J.-L. Barrat, L. Bocquet, and E. Charlaix, "Low-friction flows of liquid at nanopatterned interfaces," Nature Materials, vol. 2, no. 4, pp. 237-240, 2003.

[42] G. Whyman, E. Bormashenko, and T. Stein, "The rigorous derivation of Young, Cassie-Baxter and Wenzel equations and the analysis of the contact angle hysteresis phenomenon," Chemical Physics Letters, vol. 450, no. 4-6, pp. 355-359, 2008.

[43] F. Bottiglione, G. Carbone, and B. N. J. Persson, "Fluid contact angle on solid surfaces: Role of multiscale surface roughness," The Journal of Chemical Physics, vol. 143, no. 13, p. 134705, 2015.

[44] P. Huang and K. S. Breuer, "Direct measurement of slip length in electrolyte solutions," Physics of Fluids, vol. 19, p. 028104, 2007.

[45] H. Wu, Z. Yang, B. Cao et al., "Wetting and dewetting transitions on submerged superhydrophobic surfaces with hierarchical structures," Langmuir, vol. 33, no. 1, pp. 407-416, 2017.

[46] J. Cui, Q. Sang, Y. Li, C. Yin, Y. Li, and M. Dong, "Liquid permeability of organic nanopores in shale: calculation and analysis," Fuel, vol. 202, pp. 426-434, 2017.

[47] H. Wang, Y. Su, Z. Zhao, W. Wang, G. Sheng, and S. Zhan, "Apparent permeability model for shale oil transport through elliptic nanopores considering wall-oil interaction," Journal of Petroleum Science and Engineering, vol. 176, pp. 1041-1052, 2019.

[48] H. Fan, H. Li, and H. Wang, "Enhanced oil flow model coupling fractal roughness and heterogeneous wettability," Fractals, p. $1950088,2019$.

[49] S. Kelly, M. T. Balhoff, and C. Torres-Verdin, "Quantification of bulk solution limits for liquid and interfacial transport in nanoconfinements," Langmuir, vol. 31, no. 7, pp. 2167-2179, 2015.

[50] T. Zhang, X. Li, Z. Sun et al., "An analytical model for relative permeability in water-wet nanoporous media," Chemical Engineering Science, vol. 174, pp. 1-12, 2017.

[51] R. J. Mashl, S. Joseph, N. R. Aluru, and E. Jakobsson, "Anomalously immobilized water: a new water phase induced by confinement in nanotubes," Nano Letters, vol. 3, no. 5, pp. 589-592, 2003.

[52] S. Wang, F. Javadpour, and Q. Feng, "Fast mass transport of oil and supercritical carbon dioxide through organic nanopores in shale," Fuel, vol. 181, pp. 741-758, 2016.

[53] G. Tocci, L. Joly, and A. Michaelides, "Friction of water on graphene and hexagonal boron nitride from $\mathrm{Ab}$ initio methods: very different slippage despite very similar interface structures," Nano Letters, vol. 14, no. 12, pp. 6872-6877, 2014.

[54] M. D. Ma, L. Shen, J. Sheridan, J. Z. Liu, C. Chen, and Q. Zheng, "Friction of water slipping in carbon nanotubes," Physical Review E, vol. 83, no. 3 Pt 2, p. 036316, 2011.

[55] T. Ala-Nissila, R. Ferrando, and S. C. Ying, "Collective and single particle diffusion on surfaces," Advances in Physics, vol. 51, no. 3, pp. 949-1078, 2002.

[56] D. A. Reed and G. Ehrlich, "Surface diffusion, atomic jump rates and thermodynamics," Surface Science, vol. 102, no. 2-3, pp. 588-609, 1981.

[57] R. Gomer, "Diffusion of adsorbates on metal surfaces," Reports on Progress in Physics, vol. 53, no. 7, pp. 917-1002, 1990.

[58] E. Stefánsson and T. Loftsson, "The Stokes-Einstein equation and the physiological effects of vitreous surgery," Acta Ophthalmologica Scandinavica, vol. 84, no. 6, pp. 718-719, 2006.

[59] J. Lee, Y.-J. Yoon, J. K. Eaton, K. E. Goodson, and S. J. Bai, "Analysis of oxide $\left(\mathrm{Al}_{2} \mathrm{O}_{3}, \mathrm{CuO}\right.$, and $\left.\mathrm{ZnO}\right)$ and $\mathrm{CNT}$ nanoparticles disaggregation effect on the thermal 
conductivity and the viscosity of nanofluids," International Journal of Precision Engineering and Manufacturing, vol. 15, no. 4, pp. 703-710, 2014.

[60] S. I. Ezhenkova and S. A. Chivilikhin, "Mathematical modeling of sedimentation process of nanoparticles in gradient medium," Journal of Physics: Conference Series, vol. 643, no. 012111, 2015.

[61] C. Crank, The Mathematic of Diffusion, Clarendon Press, 1975.

[62] L. Mi, H. Jiang, and J. Li, "The impact of diffusion type on multiscale discrete fracture model numerical simulation for shale gas," Journal of Natural Gas Science and Engineering, vol. 20, pp. 74-81, 2014.

[63] S. Gisladottir, T. Loftsson, and E. Stefansson, "Diffusion characteristics of vitreous humor and saline solution follow the Stokes Einstein equation," Graefe's Archive for Clinical and Experimental Ophthalmology, vol. 247, no. 12, pp. 1677-1684, 2009.

[64] T. Zhang, X. Li, J. Shi, Z. Sun, and Y. Yin, K. Wu, J. Li, and D. Feng, "An apparent liquid permeability model of dualwettability nanoporous media: a case study of shale," Chemical Engineering Science, vol. 187, pp. 280-291, 2018.

[65] C. A. Fuentes, G. Brughmans, L. Q. N. Tran, C. Dupont-Gillain, and I. Verpoest, A. Van Vuure, "Mechanical behaviour and practical adhesion at a bamboo composite interface: physical adhesion and mechanical interlocking," Composites Science and Technology, vol. 109, pp. 40-47, 2015.

[66] C. W. Extrand, "Comment on "solid-liquid work of adhesion", Langmuir, vol. 33, no. 36, pp. 9241-9242, 2017.

[67] D. Surblys, F. Leroy, Y. Yamaguchi, and F. Müller-Plathe, "Molecular dynamics analysis of the influence of Coulomb and van der Waals interactions on the work of adhesion at the solid-liquid interface," The Journal of Chemical Physics, vol. 148, no. 13, p. 134707, 2018.

[68] K. Wu C. Zhao, and C. He, "A simple corresponding-states group-contribution method for estimating surface tension of ionic liquids," Fluid Phase Equilibria, vol. 328, pp. 42-48, 2012.

[69] E. A. Guggenheim, "The principle of corresponding states," The Journal of Chemical Physics, vol. 13, no. 7, pp. 253-261, 1945.

[70] R. Sedev, "Surface tension, interfacial tension and contact angles of ionic liquids," Current Opinion in Colloid \& Interface Science, vol. 16, no. 4, pp. 310-316, 2011.

[71] D. Feng, X. Li, X. Wang et al., "Capillary filling of confined water in nanopores: coupling the increased viscosity and slippage," Chemical Engineering Science, vol. 186, pp. 228-239, 2018.

[72] E. Bittoun and A. Marmur, "The role of multiscale roughness in the lotus effect: is it essential for super-hydrophobicity?", Langmuir, vol. 28, no. 39, pp. 13933-13942, 2012.

[73] Q. Zeng and S. Xu, "Thermodynamics and characteristics of heterogeneous nucleation on fractal surfaces," The Journal of Physical Chemistry C, vol. 119, no. 49, pp. 27426-27433, 2015.

[74] J. Cai, E. Perfect, C.-L. Cheng, and X. Hu, "Generalized modeling of spontaneous imbibition based on Hagen-Poiseuille flow in tortuous capillaries with variably shaped apertures," Langmuir, vol. 30, no. 18, pp. 5142-5151, 2014. 


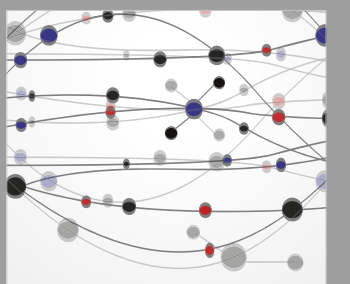

The Scientific World Journal
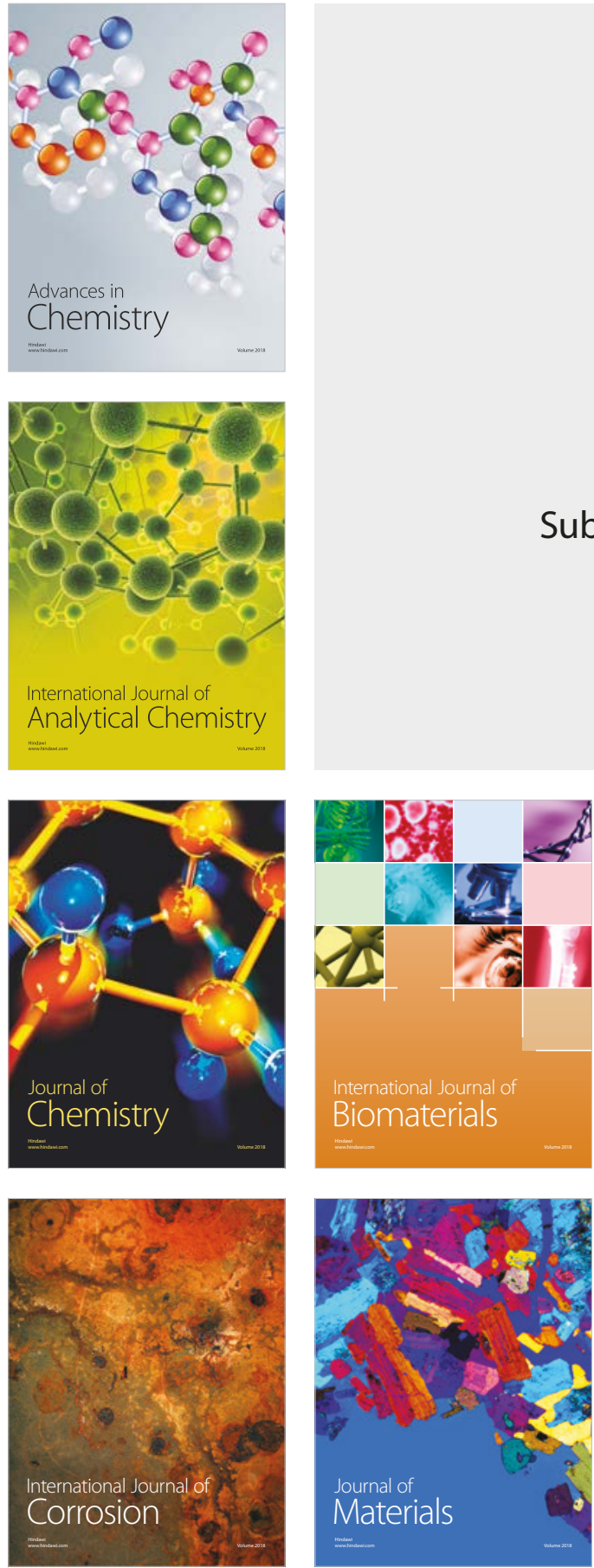

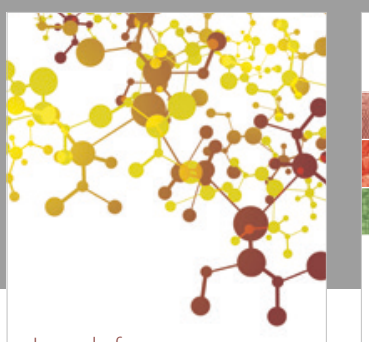

Journal of

Applied Chemistry
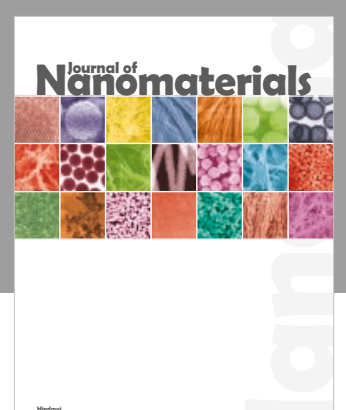

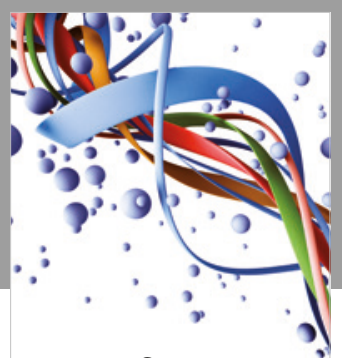

Scientifica

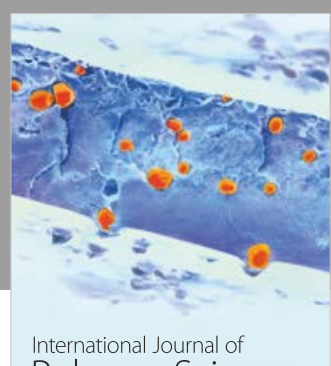

Polymer Science

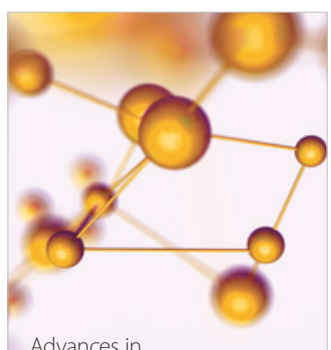

Physical Chemistry
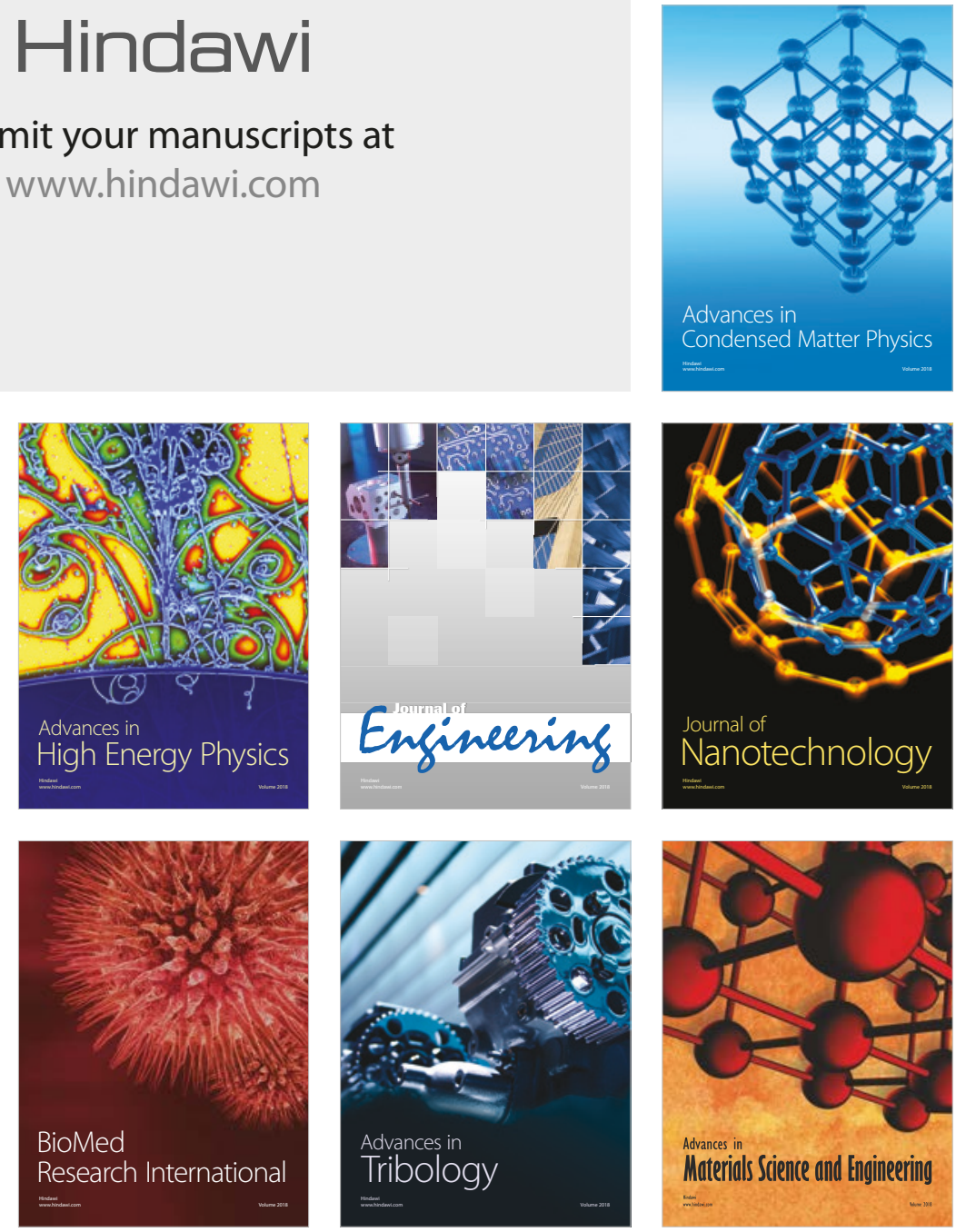